\title{
DISCRIMINATOR VARIETIES OF BOOLEAN ALGEBRAS WITH RESIDUATED OPERATORS
}

\author{
PETER JIPSEN \\ Department of Mathematics, Iowa State University \\ 400 Carver Hall, Ames, Iowa 50011, U.S.A. \\ E-mail: PJIPSEN@IASTATE.EDU
}

\begin{abstract}
The theory of discriminator algebras and varieties has been investigated extensively, and provides us with a wealth of information and techniques applicable to specific examples of such algebras and varieties.

Here we give several such examples for Boolean algebras with a residuated binary operator, abbreviated as $r$-algebras. More specifically, we show that all finite $r$-algebras, all integral $r$ algebras, all unital $r$-algebras with finitely many elements below the unit, and all commutative residuated monoids are discriminator algebras, provided they are subdirectly irreducible. These results are then used to give equational bases for some varieties of $r$-algebras. We also show that the variety of all residuated Boolean monoids is not a discriminator variety, which answers a question of B. Jónsson.
\end{abstract}

1. Preliminaries. A unary operation $f$ on a Boolean algebra $\mathbf{A}_{0}=$ $\left(A,+, 0, \cdot, 1,^{-}\right)$is additive if $f(x+y)=f(x)+f(y)$ and normal if $f(0)=0$. For an $n$-ary operation $f$ on $\mathbf{A}_{0}$, a sequence $a \in A^{n}$ and $i<n$ we define the $(\underline{a}, i)$-translate of $f$ to be the unary operation

$$
f_{\underline{a}, i}(x)=f\left(a_{0}, \ldots, a_{i-1}, x, a_{i+1}, \ldots, a_{n-1}\right) .
$$

An operator on $\mathbf{A}_{0}$ is an $n$-ary operation for which all $(\underline{a}, i)$-translates are additive and normal. Note that 0 -ary operations (constants) have no translates, so they are operators by default. $\mathbf{A}=\left(\mathbf{A}_{0}, \mathcal{F}\right)$ is a Boolean algebra with operators (BAO for short) if each $f \in \mathcal{F}$ is an operator on $\mathbf{A}_{0}$. The arity (or rank) of $f$ is denoted by $\varrho_{f}$. To be an operator on a Boolean algebra is of course an equational property, and the variety of all BAOs with operators in $\mathcal{F}$ will be denoted by $\mathrm{BAO}_{\mathcal{F}}$. The variety $\mathrm{BAO}_{\{f\}}$, where $f$ is a unary operator, is usually referred to as the variety of modal algebras (the algebraic counterpart of modal logic).

1991 Mathematics Subject Classification: Primary 06E25; Secondary 03G15, 08A40, 03B45.

The paper is in final form and no version of it will be published elsewhere. 
Although we will concern ourselves only with BAOs, the concept of a discriminator applies to algebras in general. Therefore we define it first in that context and then show how it simplifies for BAOs. In the hope of popularizing the discriminator in algebraic logic, we assume only a basic knowledge of universal algebra. A good reference for such information is [MMT].

A discriminator algebra is a nontrivial algebra $\mathbf{A}$ for which there exists a ternary term $t$ (in the language of $\mathbf{A}$ ), called a discriminator term, such that for all $x, y, z \in A$

$$
t^{\mathbf{A}}(x, x, z)=z \quad \text { and } \quad t^{\mathbf{A}}(x, y, z)=x \quad \text { if } x \neq y .
$$

The most striking consequence of the existence of such a term is that $\mathbf{A}$ must be simple, i.e., $\mathbf{A}$ admits only two congruences, namely the identity relation and the universal relation. This is because any congruence $\theta$ distinct from the identity relation identifies at least two distinct elements of $\mathbf{A}$, say $a \neq b$, so by the substitution property

$$
a=t^{\mathbf{A}}(a, b, c) \theta t^{\mathbf{A}}(a, a, c)=c
$$

for any element $c \in A$, and now it follows from transitivity that $\theta$ must be the universal relation.

In general it is not true that every simple algebra is a discriminator algebra, though we will show below that for atomic $r$-algebras (in fact for Boolean algebras with at least one atom and finitely many residuated operators) this is indeed the case.

A discriminator variety is a variety generated by a class of (similar) algebras which are discriminator algebras with respect to the same term $t$. Discriminator varieties have nice structural properties. For example,

(I) the concepts of an algebra being simple, subdirectly irreducible or directly indecomposable are equivalent in discriminator varieties,

(II) for a variety $\mathcal{V}$ and a term $t$ in the language of $\mathcal{V}$, the statement " $t$ is a discriminator term in the subdirectly irreducible members of $\mathcal{V}$ " can be characterised by equations, and

(III) in a discriminator variety $\mathcal{V}$ any universal sentence can be translated into an equation such that the sentence holds in all simple members of $\mathcal{V}$ if and only if the corresponding equation holds in $\mathcal{V}$.

In the next section we consider these results in more detail for BAOs. A good survey of discriminator algebras in general can be found in the monograph [W78] and more recent results relevant to algebraic logic are contained in [BP89]. Here we only note that for an algebra $\mathbf{A}$ that has a Boolean algebra reduct $\mathbf{A}_{0}=(A,+, 0, \cdot 1,-), \mathbf{A}$ is a discriminator algebra if and only if there exists a unary term $c$, called a unary discriminator, such that

$$
c^{\mathbf{A}}(0)=0 \quad \text { and } \quad c^{\mathbf{A}}(x)=1 \text { if } x \neq 0 .
$$


This follows from the observation that in a Boolean algebra $c$ and $t$ are interdefinable:

$$
c(x)=t(0, x, 1)^{-} \quad \text { and } \quad t(x, y, z)=x c(x \oplus y)+z c(x \oplus y)^{-},
$$

where $x \oplus y=x y^{-}+x^{-} y$ denotes the operation of symmetric difference. We use the symbol $c$ because $c^{\mathbf{A}}$ is a monadic closure operator on $\mathbf{A}_{0}$. For example, any relation algebra $\left(\mathbf{A}_{0}, ;,{ }^{\prime}, 1^{\prime}\right)$ is a discriminator algebra with unary discriminator term $c(x)=1 ; x ; 1$. Much of the subsequent material addresses the question which generalisations of relation algebras are discriminator algebras.

A unary operation on a Boolean algebra $\mathbf{A}_{0}$ is residuated if there exists a residual operation $g$ such that for all $x, y \in A$

$$
f(x) \leq y \quad \text { iff } \quad x \leq g(y) .
$$

Equivalently, $f$ is residuated if there exists a conjugate operation $h$ such that for all $x, y \in A$

$$
f(x) y=0 \quad \text { iff } \quad x h(y)=0 .
$$

If they exist, then $g$ and $h$ are unique, and they are related by the formulas $h(x)=g\left(x^{-}\right)^{-}$and $g(x)=h\left(x^{-}\right)^{-}$. The operation $f$ is selfconjugate if it is equal to its conjugate.

Note that the relation "is a conjugate of" is symmetric. This is one of the reasons why it is more convenient to consider conjugates instead of residuals. However, the concept of residuation applies more generally to posets, and is also known as Galois connection or adjunction in category theory.

The following simple but important observations about residuated operations in Boolean algebras appeared in [JT51]:

(IV) Every residuated operation is normal and completely additive (i.e. preserves all existing joins).

(V) $f$ and $h$ are conjugate operations on $\mathbf{A}_{0}$ if and only if they are normal and for all $x, y \in A$

$$
f(x) y \leq f(x h(y)) \quad \text { and } \quad x h(y) \leq h(f(x) y) .
$$

Two $n$-ary operations $f$ and $h$ on $\mathbf{A}$ are conjugate in the $i$-th argument if $f_{\underline{a}, i}$ is conjugate to $h_{a, i}$ for all $\underline{a} \in A^{n}$.

Let $\mathbf{A}=\left(\mathbf{A}_{0}, \mathcal{F}\right)$ be a Boolean algebra with additional operations. We say that $\mathbf{A}$ is a Boolean algebra with residuated operators (residuated $\mathrm{BAO}$ for short) if for each nonconstant $f \in \mathcal{F}$ and all $i<\varrho_{f}$ there exist a $\varrho_{f}$-ary term $t$ which is conjugate to $f$ in the $i$ th argument. It follows immediately from (IV) that $\mathbf{A} \in \mathrm{BAO}_{\mathcal{F}}$. Again we mention relation algebras $\mathbf{A}=\left(\mathbf{A}_{0}, ;,{ }^{\prime}, 1^{\prime}\right)$ as motivating examples of residuated BAOs, since ${ }^{\sim}$ is selfconjugate and ; has conjugate terms

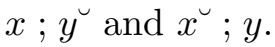

An $r$-algebra is a Boolean algebra $\mathbf{A}_{0}$ with three residuated binary operations $\circ, \triangleright$ and $\triangleleft$, where $\triangleright$ and $\triangleleft$ are the right and left conjugates of $\circ$, i.e., the conditions

$$
(x \circ y) z=0, \quad(x \triangleright z) y=0 \quad \text { and } \quad(z \triangleleft y) x=0
$$


are equivalent. By $(\mathrm{V})$ this is an equational property, whence it follows that the class of all $r$-algebras is a (finitely based) variety.

A unital $r$-algebra or ur-algebra is of the form $\left(\mathbf{A}_{0}, \circ, \triangleright, \triangleleft, e\right)$, where $e$ is a unit element with respect to $\circ$ (i.e. $e \circ x=x \circ e=x$ for all $x \in A$ ). A residuated Boolean monoid, or $r m$-algebra, is a ur-algebra in which the operation $\circ$ is associative.

A motivation for studying $r$-algebras is that they are natural generalisations of relation algebras: for any relation algebra $\mathbf{A}=\left(\mathbf{A}_{0}, ;,^{\smile}, 1^{\prime}\right)$ we obtain an $\mathrm{rm}$ -

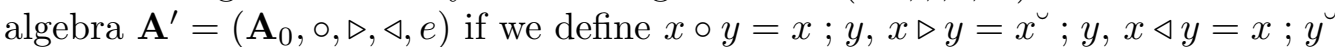
and $e=1$ '. If we consider nonassociative relation algebras, as in [Ma82], then we obtain ur-algebras. Conversely, it is shown in [JTs91] that if a ur-algebra satisfies the equations $x \triangleright y=(x \triangleright e) \circ y$ and $x \triangleleft y=x \circ(y \triangleright e)$ then it is (term-definably equivalent to) a nonassociative relation algebra, with $x^{\longleftarrow}=x \triangleright e$.

Other areas where $r$-algebras occur are in the theory of automata as complex algebras over monoids, in axiomatic treatments of the betweenness relation in geometry, in the algebraic study of relevance logics (with classical negation), and in the study of complex algebras of ternary relational structures in general. Residuated BAOs have also been studied in a more category-theoretical setting in [GM90].

2. Discriminator varieties of Boolean algebras with operators. Since Boolean algebras are term-definably equivalent to Boolean rings, Boolean congruence relations are determined by their 0-congruence classes or ideals. In particular, if $I$ is an ideal of $\mathbf{A}_{0}$ then the corresponding Boolean congruence relation is given by

$$
x \theta_{I} y \quad \text { iff } \quad x \oplus y \in I .
$$

An ideal of a BAO is a congruence ideal if it is the 0-congruence class of some congruence relation on the algebra. The following result gives an internal characterisation of congruence ideals.

Lemma 1. Let $\mathbf{A} \in \mathrm{BAO}_{\mathcal{F}}$. For a Boolean ideal I of $\mathbf{A}_{0}$ the following are equivalent:

(i) $I$ is a congruence ideal of $\mathbf{A}$,

(ii) $x \in I$ implies $f_{\underline{1}, i}(x) \in I$ for all $f \in \mathcal{F}$ and $i<\varrho_{f}$, where $\underline{1}$ is a sequence of 1 's of length $\varrho_{f}$.

Pro of. If (i) holds then $\theta_{I}$ is a congruence on $\mathbf{A}$, so for all $x \in I, f \in \mathcal{F}$ and $i<\varrho_{f}$

$$
x \theta_{I} 0 \Rightarrow f_{\underline{1}, i}(x) \theta_{I} f_{\underline{1}, i}(0)=0 \Rightarrow f_{\underline{1}, i}(x) \in I .
$$

Conversely, suppose (ii) holds. We have to show that $\theta_{I}$ has the substitution property for all $f \in \mathcal{F}$. Since $\theta_{I}$ is transitive, it suffices to show that for all $f \in \mathcal{F}$, all $i<\varrho_{f}$ and all $\underline{a} \in A^{\varrho_{f}}$

$$
x \theta_{I} y \text { implies } f_{\underline{a}, i}(x) \theta_{I} f_{\underline{a}, i}(y) .
$$


So suppose $x \oplus y=x y^{-}+x^{-} y \in I$. By the additivity of $f$ we have

$$
f_{\underline{a}, i}(x) f_{\underline{a}, i}(y)^{-}=f_{\underline{a}, i}(x y) f_{\underline{a}, i}(y)^{-}+f_{\underline{a}, i}\left(x y^{-}\right) f_{\underline{a}, i}(y)^{-} \leq 0+f_{\underline{1}, i}\left(x y^{-}\right) \in I .
$$

Similarly $f_{\underline{a}, i}(x)^{-} f_{\underline{a}, i}(y) \in I$, whence the result follows.

For an element $a$ in a BAO A let $A a=\{x a: x \in A\}=\{y \in A: y \leq a\}$ be the principal ideal generated by $a$, and let $\mathbf{A}_{0} a$ be the relativised Boolean algebra $\left(A a,+, 0, \cdot, 1,{ }^{-a}\right)$ with relative complement $x^{-a}=x^{-} a$. The relativised BAO $\mathbf{A} a$ is defined to be $\left(\mathbf{A}_{0} a,\left\{f_{a}: f \in \mathcal{F}\right\}\right)$ where $f_{a}(\underline{x})=f(\underline{x}) a$ for any sequence $\underline{x} \in(A a)^{\varrho_{f}}$.

A congruence element in a BAO $\mathbf{A}$ is an element $a \in A$ for which the principal ideal $A a$ is a congruence ideal. By Lemma 1, $a$ is a congruence element if and only if

$$
f_{\underline{1}, i}(a) \leq a \quad \text { for all } f \in \mathcal{F} \text { and } i<\varrho_{f} .
$$

Note that the map $\varphi(x)=x a$ is a Boolean homomorphism from $\mathbf{A}_{0}$ to $\mathbf{A}_{0} a$, with kernel $A a^{-}$. Therefore $\varphi$ is a BAO homomorphism from $\mathbf{A}$ to $\mathbf{A} a$ if and only if $a^{-}$is a congruence element. A universal algebraic result about factor congruences can now be stated in this context as follows:

Theorem 2. A BAO A can be decomposed into a direct product of two nontrivial factors if and only if there exists $a \in A$ such that $a \notin\{0,1\}$ and both $a$ and $a^{-}$are congruence elements of $\mathbf{A}$. In this case $\mathbf{A} \cong \mathbf{A} a \times \mathbf{A} a^{-}$.

We now apply these observations to discriminator varieties. The following theorem is a reformulation for BAOs of McKenzie's characterisation of discriminator varieties in [M75] (cf. (II) above).

TheOREM 3. Let $\mathcal{V}$ be a subvariety of $\mathrm{BAO}_{\mathcal{F}}$ and let $c$ be a unary term of $\mathcal{V}$. The following are equivalent:

(i) $c$ is a unary discriminator in all subdirectly irreducible members of $\mathcal{V}$,

(ii) $\mathcal{V}$ satisfies the equations $c(0)=0, x \leq c(x)$,

$$
f_{\underline{1}, i}(c(x)) \leq c(x) \quad \text { and } \quad f_{\underline{1}, i}\left(c(x)^{-}\right) \leq c(x)^{-}
$$

for each operator $f \in \mathcal{F}$ and $i<\varrho_{f}$.

Proof. (i) $\Rightarrow$ (ii). By definition $c^{\mathbf{A}}(0)=0, c^{\mathbf{A}}(x)=1$ if $x \neq 0$, and $f_{\underline{1}, i}^{\mathbf{A}}(0)=0$ for each $f \in \mathcal{F}$ and $i<\varrho_{f}$, so the equations hold in $\mathcal{V}$.

(ii) $\Rightarrow$ (i). Let $\mathbf{A}$ be a subdirectly irreducible algebra in $\mathcal{V}$. By Lemma 1 the equations $(*)$ imply that $c^{\mathbf{A}}(x)$ and $c^{\mathbf{A}}(x)^{-}$are congruence elements for every $x \in A$. Since any subdirectly irreducible member of $\mathcal{V}$ is directly indecomposable, Theorem 2 implies that $c^{\mathbf{A}}(x)$ is either 0 or 1 . For $x \neq 0$ the equation $x \leq c(x)$ implies that $c^{\mathbf{A}}(x) \neq 0$, so $c^{\mathbf{A}}(x)=1$.

Observe that in a residuated $\mathrm{BAO}$ the complement of a congruence element is also a congruence element, since if $t$ is a conjugate term for $f \in \mathcal{F}$ in the $i$ th argument then $t_{\underline{1}, i}$ is normal and hence $t_{\underline{1}, i}(a) \leq a$ for any congruence element $a$, 
which is equivalent to $t_{\underline{1}, i}(a) a^{-}=0, f_{\underline{1}, i}\left(a^{-}\right) a=0$ and finally $f_{\underline{1}, i}\left(a^{-}\right) \leq a^{-}$. Therefore half the equations $(*)$ are redundant in this case. Also, the existence of a nontrivial congruence element in a residuated BAO implies that the algebra is decomposable. Since every ideal in a finite Boolean algebra is necessarily principal, it follows that for finite residuated BAOs the properties of being indecomposable, subdirectly irreducible and simple are equivalent.

For a $\mathrm{BAO} \mathbf{A}=\left(\mathbf{A}_{0}, \mathcal{F}\right)$ of finite similarity type (i.e. $\mathcal{F}$ finite), define the term $\tau$ by

$$
\tau(x)=\sum\left\{f_{\underline{1}, i}(x): f \in \mathcal{F}, 0<\varrho_{f}, i<\varrho_{f}\right\} .
$$

So for example in a $u r$-algebra $\left(\mathbf{A}_{0}, \circ, \triangleright, \triangleleft, e\right)$

$$
\tau(x)=1 \circ x+x \circ 1+1 \triangleright x+x \triangleleft 1+1 \triangleleft x+x \triangleright 1 .
$$

It now follows from Lemma 1 that $\mathbf{A}$ and the modal algebra $\left(\mathbf{A}_{0}, \tau^{A}\right)$ have identical congruence lattices.

With this notation we can also summarise the equations $(*)$ as

$$
\tau(c(x)) \leq c(x) \text { and } \tau\left(c(x)^{-}\right) \leq c(x)^{-} .
$$

Note that for $r$-algebras $\tau(x)$ is selfconjugate and so the two equations are equivalent.

TheOrem 4. Let $\mathbf{A} \in \mathrm{BAO}_{\mathcal{F}}$ be of finite type. If $\mathbf{A}$ is simple, contains at least one atom, and if $\tau(x)$ is selfconjugate then $\mathbf{A}$ is a discriminator algebra with unary discriminator $c(x)=\tau^{n}(x)$ for some $n \in \omega$.

Proof. For any atom $a \in A$, the congruence ideal generated by $a$ is the join of all principal ideals $A \tau^{n}(a), n \in \omega$. If $\mathbf{A}$ is simple, then this join must be $A$, which is a compact congruence ideal of $\mathbf{A}$. Therefore there exists $n_{a} \in \omega$ such that $\tau^{n_{a}}(a)=1$.

Now for any nonzero $x \in A, \tau^{n_{a}}(a) x \neq 0$, hence $\tau^{n_{a}}(x) a \neq 0$ and $a \leq \tau^{n_{a}}(x)$, since $\tau$ is selfconjugate and $a$ is an atom. Consequently, $1=\tau^{n_{a}}(a) \leq \tau^{2 n_{a}}(x)$ for all nonzero $x \in A$ and therefore $c(x)=\tau^{2 n_{a}}(x)$ is a unary discriminator.

In light of the remarks after Theorem 3 we also have the following result.

COROLlaRY 5. Every finite subdirectly irreducible residuated BAO of finite type is a discriminator algebra with unary discriminator $c(x)=\tau^{n}(x)$ for some $n \in \omega$.

An $r$-algebra is integral if for all elements $x, y$ the condition $x \circ y=0$ implies $x=0$ or $y=0$. We now give an application of Theorem 3 to axiomatise the variety generated by all integral $r$-algebras.

LEMMA 6. An r-algebra $\mathbf{A}$ is integral if and only if the term $c(x)=x \triangleright 1$ is a unary discriminator for $\mathbf{A}$. 
Proof. Assume A integral and let $x$ be a nonzero element of $\mathbf{A}$. Then the conditions

$$
(x \triangleright 1) y=0, \quad x \circ y=0 \text { and } y=0
$$

are equivalent, whence $x \triangleright 1=1$. Conversely, if $x \triangleright 1$ is a unary discriminator then $x \neq 0$ and $y \neq 0$ imply $(x \triangleright 1) y \neq 0$, hence $x \circ y \neq 0$.

Corollary 7. For a variety $\mathcal{V}$ of $r$-algebras, the following are equivalent:

(i) $\mathcal{V}$ is the largest variety for which $c(x)=x \triangleright 1$ is a unary discriminator in all its subdirectly irreducible members.

(ii) $\mathcal{V}$ is generated by all integral r-algebras.

(iii) $\mathcal{V}$ is the variety of all $r$-algebras that satisfy the equation $x+\tau(x \triangleright 1) \leq x \triangleright 1$.

Proof. (i) is equivalent to (ii) by Lemma 6, and the equivalence of (i) and (iii) follows from Theorem 3 once we observe that $0 \triangleright 1=0$ and $\tau$ is selfconjugate for $r$-algebras.

We end this section with an explicit description (for BAOs) of the translation from universal sentences to equations, mentioned in (III) above. Let $\mathcal{V}$ be a discriminator variety of BAOs with unary discriminator $c(x)$ and let $\sigma$ be a universal sentence in the language of $\mathcal{V}$. Equivalently, we can view $\sigma$ as a universally quantified open formula and we may assume that it is built up from atomic formulas (i.e. equations of terms) using only conjunction and negation. Steps (A)-(C) below inductively define a term $\sigma^{*}$ of $\mathcal{V}$ such that

$$
\mathcal{V} \vDash \sigma^{*}=1 \quad \text { if and only if } \quad \operatorname{Si}(\mathcal{V}) \vDash \sigma .
$$

(A) If $\sigma$ is an atomic formula $s=t$, let $\sigma^{*}=(s \oplus t)^{-}$,

(B) if $\sigma$ is a conjuction of two open formulas $\varphi$ and $\psi$, let $\sigma^{*}=\varphi^{*} \cdot \psi^{*}$ and

(C) if $\sigma$ is the negation of an open formula $\varphi$, let $\sigma^{*}=c\left(\varphi^{*-}\right)$.

3. Discriminator $u r$-algebras. We first list some properties that hold in all ur-algebras.

LEMMA 8. For any $x, y, u, v$ in a ur-algebra with $u, v \leq e$ we have

(i) $x \leq y \circ u$ implies $x=x \circ u$,

(ii) $(x \circ u) y=(y \circ u) x$,

(iii) $x \triangleleft u=x \circ u$ and $u \triangleright x=u \circ x$,

(iv) $u \circ v=u v$,

$(\mathrm{v})(x \circ u) v=(x \triangleright u) v=(x \triangleleft u) v=(u \circ x) v=(u \triangleright x) v=(u \triangleleft x) v=x u v$,

(vi) $(1 \circ u) x=x \circ u$ and $(u \circ 1) x=u \circ x$.

Proof. (i) By assumption, $x \leq y \circ u$ implies $x \leq y$, so $y=x+y x^{-}$. Now

$$
x \leq y \circ u=\left(x+y x^{-}\right) \circ u=x \circ u+y x^{-} \circ u .
$$

But $x\left(y x^{-} \circ u\right) \leq x y x^{-}=0$, hence $x \leq x \circ u \leq x$. 
(ii) Let $z=(y \circ u) x \leq y \circ u$. Then (i) implies that $z=z \circ u \leq x \circ u$. Since we also have $z \leq y x \leq y$, we get $z=(y \circ u) x \leq(x \circ u) y$. For the reverse direction we simply interchange $x$ and $y$.

(iii) Conjugation and (ii) imply that the conditions $(x \triangleleft u) y \neq 0,(y \circ u) x \neq 0$ and $(x \circ u) y \neq 0$ are equivalent, hence $x \triangleleft u=x \circ u$.

(iv) First we note that $u=u \circ u$ since $u=u \circ e=u \circ u+u \circ \mathrm{eu}^{-}$and $u \circ e u^{-} \leq$ $u u^{-}=0$. Therefore $u v=u v \circ u v \leq u \circ v$. Conversely, $u \circ v \leq(u \circ e)(e \circ v) \leq u v$.

(v) By additivity $(x \circ u) v=(x e \circ u) v+\left(x e^{-} \circ u\right) v=x u v$ since $\left(x e^{-} \circ u\right) v \leq e^{-} e$ $=0$. By (iii) we also have $(x \triangleleft u) v=x u v$. Now $(x \triangleright u) v=(x e \triangleright u) v+\left(x e^{-} \triangleright u\right) v=$ $x e u v+0=x u v$, again using (iii).

(vi) A direct calculation gives $(1 \circ u) x=(x \circ u) x+\left(x^{-} \circ u\right) x=x \circ u$

Although there are several ways in which one might generalise the notions of domain and range of a relation for an element in a ur-algebra, we find the following one most useful. For $x \in A$ let

$$
x^{\delta}=(1 \triangleleft x) e \quad \text { and } \quad x^{\varrho}=(x \triangleright 1) e
$$

be the domain and range of $x$ respectively. With this definition, $u=e x^{\delta-}$ is the largest element below $e$ for which $u \circ x=0$ (see proof of (iii) below). We summarise some of the properties of these operations:

Lemma 9. For any $x$ in a ur-algebra

(i) $x \leq e$ implies $x^{\delta}=x=x^{\varrho}$,

(ii) $x^{\delta \delta}=x^{\delta}$ and $x^{\varrho \varrho}=x^{\varrho}$, and

(iii) $x^{\delta} \circ x=x=x \circ x^{\varrho}$.

Proof. (i) follows from Lemma $8(\mathrm{v})$, and (ii) is an immediate consequence of (i). To prove (iii), we compute $x=e \circ x=x^{\delta} \circ x+e x^{\delta-} \circ x$, and $\left(e x^{\delta-} \circ x\right) 1=0$ since $(1 \triangleleft x) e x^{\delta-}=x^{\delta} x^{\delta-}=0$.

We now prove a general result about congruence lattices of modal algebras, and then apply it to ur-algebras in which the unit element is the join of finitely many atoms.

TheOREM 10. Let $\mathbf{A}=\left(\mathbf{A}_{0}, f\right)$ be a modal algebra and suppose that $A$ satisfies the inclusion $x \leq f\left(f^{n}(x) u\right)$ for some (fixed) $u \in \mathbf{A}$ and $n \in \omega$. Then

$$
\operatorname{Con}(\mathbf{A}) \cong \operatorname{Con}\left(\mathbf{A}_{0} u, g^{\mathbf{A}}\right)
$$

where $g(x)=f^{n+2}(x) u$.

Proof. We will show that the maps

$$
F(I)=I u \quad \text { and } \quad G(J)=A f(J)=\{x \in A: x \leq f(y) \text { for some } y \in J\}
$$

map congruence ideals to congruence ideals and are inverses of each other. Since they are also order preserving, the result follows.

Note that the inclusion $x \leq f\left(f^{n}(x) u\right)$ implies $f^{2}(x) \leq f(g(x))$, and therefore (*)

$$
f^{n+1}(x) \leq f\left(g^{n}(x)\right) \text {. }
$$


For any congruence ideal $I$ of $\mathbf{A}, F(I)$ is clearly a Boolean ideal, and if $x \in$ $I u$ then $f^{n+2}(x) \in I$, hence $g(x) \in I u$. Now consider a congruence ideal $J$ of $\left(\mathbf{A}_{0} u, g^{\mathbf{A}}\right)$. Again $G(J)$ is easily seen to be a Boolean ideal. So suppose $x \in A f(J)$, whence $x \leq f(y)$ for some $y \in J$. Then

$$
f(x) \leq f^{2}(y) \leq f\left(f^{n+2}(y) u\right) \leq f(g(y))
$$

and since $g(y) \in J$ it follows that $f(x) \in A f(J)$. This shows that $F$ and $G$ map congruence ideals to congruence ideals.

Next we show that $F G(J)=J$. Let $x \in F G(J)=A f(J) u$. Then $x \leq f(y) u$ for some $y \in J$, hence

$$
x \leq f\left(f^{n}(x) u\right) u \leq f^{n+1}(x) u \leq f^{n+2}(y) u=g(y) \in J .
$$

Conversely, for any $x \in J$, we have $x \leq u$, hence $x \leq f^{n+1}(x) \leq f\left(g^{n}(x)\right)$, where the last inclusion follows from $(*)$ above.

Finally, we have $G F(I)=I$ since $A f(I u) \subseteq f(I) \subseteq I$, and for any $x \in I$, $x \leq f\left(f^{n}(x) u\right) \in f(I u)$, hence $x \in A f(I u)$.

Recall that for a $\mathrm{BAO} \mathbf{A}=\left(\mathbf{A}_{0}, \mathcal{F}\right)$ of finite type $\operatorname{Con}(\mathbf{A})$ is isomorphic to $\operatorname{Con}\left(\mathbf{A}_{0}, \tau^{\mathbf{A}}\right)$, where $\tau$ is the join of all $(\underline{1}, i)$-translates of $f \in \mathcal{F}, i<\varrho_{f}$.

Corollary 11. Suppose A is a ur-algebra, and let $g(x)=\tau^{3}(x)$ e. Then

$$
\operatorname{Con}(\mathbf{A}) \cong \operatorname{Con}\left(\mathbf{A}_{0} e, g^{\mathbf{A}}\right) .
$$

Proof. The result will follow from the previous theorem with $f(x)=\tau(x)$ and $n=2$ once we establish the inclusion $x \leq \tau(\tau(x) e)$. But this is immediate, since by Lemma 9(iii), $x=x^{\delta} \circ x \leq(1 \triangleleft x) e \circ 1 \leq \tau(\tau(x) e)$.

COROllary 12. Any subdirectly irreducible ur-algebra $\mathbf{A}$ in which the identity element $e$ is the join of finitely many atoms is a discriminator algebra.

Proof. In this case the algebra $\left(\mathbf{A}_{0} e, g^{\mathbf{A}}\right)$ from the preceding corollary is a finite subdirectly irreducible selfconjugate modal algebra. By Corollary 5 it is a discriminator algebra and therefore simple. But then $\mathbf{A}$ is also simple by Corollary 11, whence Theorem 4 implies that it is a discriminator algebra.

If $e$ is an atom of $\mathbf{A}$ then one can easily show by a direct calculation that $c(x)=(1 \triangleright x) \circ 1$ is a unary discriminator. Note also that $e$ is an atom of $\mathbf{A}$ if and only if $\mathbf{A}$ satisfies the universal sentence

$$
\text { for all } x \in A \text { either } e x=0 \text { or } e x^{-}=0 \text {. }
$$

Using steps (A)-(C) at the end of Section 2 to translate this into an equation and combining it with Theorem 3 we obtain the following result:

THEOREM 13. Let $\mathcal{V}_{e}$ be the variety generated by all ur-algebras in which $e$ is an atom, and let $c(x)=(1 \triangleright x) \circ 1$. Then the following equations form a basis for $\mathcal{V}_{e}$ relative to the variety of all ur-algebras:

$$
x+\tau(c(x)) \leq c(x) \quad \text { and } \quad c\left(c(e x)^{-}+c\left(e x^{-}\right)^{-}\right)=1 .
$$


Note that $e$ is an atom in any integral ur-algebra (by Lemma 8(iv)) but, in contrast to relation algebras, the converse does not hold in general.

We now turn to $\mathrm{rm}$-algebras, i.e. ur-algebras that are associative with respect to $\circ$. The main result (Corollary 17) is that the variety of commutative rmalgebras is a subvariety of $\mathcal{V}_{e}$ above.

Lemma 14. For any $x, u, v$ in an rm-algebra with $u, v \leq e$ we have

(i) $(1 \circ u)(1 \circ v)=1 \circ u v$ and

(ii) $(x \circ u)(y \circ v)=x y \circ u v$.

Proof. (i) $(1 \circ u)(1 \circ v)=1 \circ v \circ u=1 \circ u v$ by Lemma $8(i i)$, (iv) and associativity.

(ii) Using (i) and Lemma $8(v i)$ we calculate $(x \circ u)(y \circ v)=(1 \circ u)(1 \circ v) x y=$ $(1 \circ u v) x y=x y \circ u v$.

The next lemma gives several equivalent formulations of the associative law in $r$-algebras. A general method for proving such equivalences in residuated BAOs is given in [JTs91], although they can also be verified by direct computation.

LEMMA 15. The following identities are equivalent in any r-algebra:

(i) $(x \circ y) \circ z=x \circ(y \circ z)$,

(ii) $(x \triangleright y) \triangleleft z=x \triangleright(y \triangleleft z)$,

(iii) $(x \circ y) \triangleright z=y \triangleright(x \triangleright z)$,

(iv) $x \triangleleft(y \circ z)=(x \triangleleft z) \triangleleft y$.

Note also that the commutative law $x \circ y=y \circ x$ is equivalent to $x \triangleright y=y \triangleleft x$.

TheOrem 16. Let $\mathbf{A}=\left(\mathbf{A}_{0}, \circ, \triangleright, \triangleleft, e\right)$ be a commutative rm-algebra. Then $1 \circ u$ is a congruence element for any $u \leq e$.

Pr o of. Let $x=1 \circ u$. Since $\mathbf{A}$ is commutative, it suffices to show that $1 \circ x \leq x$, $1 \triangleright x \leq x$ and $1 \triangleleft x \leq x$. By associativity $1 \circ x=x$. By Lemma $8($ iii), $x=1 \triangleleft u$, so

$$
1 \triangleright x=1 \triangleright(1 \triangleleft u)=(1 \triangleright 1) \triangleleft u=1 \triangleleft u=x
$$

by Lemma 15(ii). Finally, $1 \triangleleft x=1 \triangleleft(1 \circ u)=(1 \triangleleft u) \triangleleft 1$ using Lemma 15(iv). By commutativity $(1 \triangleleft u) \triangleleft 1=1 \triangleright(1 \triangleleft u)=x$ as before.

COROLlaRY 17. The variety of all commutative rm-algebras is a discriminator variety, and in the simple members e is an atom.

Proof. Suppose A is a commutative $r m$-algebra in which $e$ is not an atom. Then there exists a nonzero element $u \leq e$ such that $v=e u^{-}$is also nonzero. By the preceding theorem $u \circ 1$ and $v \circ 1$ are congruence elements, and by Lemma 14 they are disjoint, so it follows from the additivity of o that they are complements of each other. Therefore $\mathbf{A}$ is directly decomposable by Theorem 2. As a result $e$ is necessarily an atom in any subdirectly irreducible commutative $r m$-algebra, and hence the variety of all commutative $r m$-algebras is a subvariety of $\mathcal{V}_{e}$. By Corollary $12, \mathcal{V}_{e}$ is a discriminator variety, and this property carries over to all its subvarieties. 
The previous result generalises an unpublished result of C. Tsinakis, who proved that the variety of commutative Euclidean (defined below) $\mathrm{rm}$-algebras is a discriminator variety.

4. Categories, Euclidean $r m$-algebras and a counterexample. Here we show that certain $r m$-algebras can be constructed as complex algebras of (small) categories. We then use this observation to show that, in spite of the many examples of discriminator algebras and varieties of residuated BAOs in the previous sections, the variety of all $\mathrm{rm}$-algebras is not a discriminator variety.

At the end of Section 1 we remarked that $r$-algebras occurred in the algebraic study of ternary relational structures. We now elaborate on this remark. Let $\mathbf{U}$ be a relational structure $(U, R)$ for some ternary relation $R \subseteq U^{3}$. The complex algebra of $\mathbf{U}$ is defined as $\mathbf{U}^{+}=\left(U^{+}, R^{+}\right)$, where $U^{+}$is the Boolean algebra of sets $\left(\mathcal{P}(U), \cup, \emptyset, \cap, U^{-}\right)$and for $X, Y \subseteq U$

$$
R^{+}(X, Y)=\{z \in U:(x, y, z) \in R \text { for some } x \in X, y \in Y\} .
$$

It is easy to see that $\mathbf{U}^{+}$is a BAO, but note that the definition of $R^{+}$treats the last coordinate of $R$-tuples in a special way. We can just as well define two other operations

$$
\begin{aligned}
& R_{1}^{+}(X, Z)=\{y \in U:(x, y, z) \in R \text { for some } x \in X, z \in Z\} \quad \text { and } \\
& R_{0}^{+}(Z, Y)=\{x \in U:(x, y, z) \in R \text { for some } y \in Y, z \in Z\} .
\end{aligned}
$$

Now the residuated complex algebra of $\mathbf{U}$, denoted by $\mathbf{U}^{\oplus}$, is defined as $\left(U^{+}, R^{+}, R_{1}^{+}, R_{0}^{+}\right)$and, when it is clear from the context what relation $R$ we are working with, we denote $R^{+}, R_{1}^{+}, R_{0}^{+}$by $\circ, \triangleright, \triangleleft$ respectively. Again it is easy to see that $\mathbf{U}^{\oplus}$ is a residuated BAO and thus an $r$-algebra. The concepts of complex algebra and residuated complex algebra can, of course, be defined for relational structures of arbitrary type, but this is not needed here.

Every $r$-algebra can be embedded in the residuated complex algebra of a ternary relational structure. In the general case this result is known as the representation theorem for BAOs and is a straightforward consequence of the (nontrivial) result that every $\mathrm{BAO}$ can be embedded in its canonical extension, which is a complete and atomic BAO. This embedding preserves equations that contain no complementation, so the canonical extension of a residuated BAO is again residuated. For a recent treatment of these classical results of [JT51] see [J91].

We aim to construct an $r m$-algebra that is not a discriminator algebra, so we need to consider some specialised relational structures. A partial semigroup is a structure $(U, \circ)$ where $\circ$ is a partial binary operation on $U$ such that whenever $a \circ b$ and $(a \circ b) \circ c$ are defined, then $b \circ c$ and $a \circ(b \circ c)$ are defined, and conversely, and $(a \circ b) \circ c=a \circ(b \circ c)$. Note that the residuated complex algebra of a partial semigroup is an associative $r$-algebra, with $\circ$ defined on subsets of $U$ in the usual way by

$$
X \circ Y=\{x \circ y: x \in X \text { and } y \in Y\}
$$


A (small) category is a special kind of partial semigroup with a distinguished subset $E \subseteq U$ (of identity morphisms) such that $E \circ x=x \circ E=x$. Thus the residuated complex algebra of a category is an $r m$-algebra. But we get somewhat more since a category also has to satisfy the property: whenever $a \circ b$ and $b \circ c$ are defined, then $(a \circ b) \circ c$ (and $a \circ(b \circ c))$ is defined (see [MMT]). This suggests considering the following property for $r$-algebras:

$$
a \circ b \neq 0 \neq b \circ c \text { implies }(a \circ b) \circ c \neq 0 \neq a \circ(b \circ c) .
$$

An $r$-algebra that satisfies this property will be called weakly Euclidean.

An $r$-algebra is said to be Euclidean if it satisfies the inclusion $(a \triangleright b) \circ c \leq$ $a \triangleright(b \circ c)$ or equivalently if

$$
(a \triangleright x)(y \triangleleft c) \neq 0 \quad \text { implies } \quad(a \circ y)(x \circ c) \neq 0 .
$$

An $r$-algebra is said to be strongly Euclidean if it satisfies the identity $(a \triangleright b) \circ c=$ $a \triangleright(b \circ c)$. The implicational form of the Euclidean inclusion appeared in [P61] in the context of a modern treatment of geometry. Every relation algebra is strongly Euclidean, and it is shown in [JTs91] that for ur-algebras the converse is also true. The following lemma establishes a connection between Euclidean and weakly Euclidean $r$-algebras. Note that $\operatorname{At}(\mathbf{A})$ is the set of atoms of the Boolean reduct of $\mathbf{A}$.

LEMMA 18. Let A be an r-algebra.

(i) If $\mathbf{A}$ is Euclidean then it is weakly Euclidean.

(ii) If $\mathbf{A}$ is atomic, weakly Euclidean and $(\operatorname{At}(\mathbf{A}), \circ)$ is a partial semigroup then $\mathbf{A}$ is Euclidean.

Proof. (i) Since the Euclidean inclusion contains no complementation, it is preserved under canonical extensions, so we may assume that $\mathbf{A}$ is atomic. Consider $a, b, c \in \operatorname{At}(\mathbf{A})$. If $a \circ b \neq 0$ then $(a \triangleright(a \circ b)) b \neq 0$, hence $(a \triangleright(a \circ b)) \geq b$, and similarly if $b \circ c \neq 0$ then $((b \circ c) \triangleleft c) \geq b$. Therefore $(a \triangleright(a \circ b))((b \circ c) \triangleleft c) \neq 0$ and, since $\mathbf{A}$ is Euclidean, $(a \circ(b \circ c))((a \circ b) \circ c) \neq 0$. So the weakly Euclidean property holds for all atoms of $\mathbf{A}$, and by additivity it extends to all of $\mathbf{A}$.

(ii) Suppose $(a \triangleright x)(y \triangleleft c) \neq 0$ for some $a, x, y, c \in \operatorname{At}(\mathbf{A})$. Then there exists an atom $b \in A$ such that $b \leq a \triangleright x$ and $b \leq y \triangleleft c$, or equivalently $x \leq a \circ b$ and $y \leq b \circ c$. By assumption $\circ$ is a partial operation, so we actually have $x=a \circ b$ and $y=b \circ c$. From the associative law it follows that $x \circ c=a \circ b \circ c=a \circ y$. Now, $\mathbf{A}$ is assumed to be weakly Euclidean, hence $0 \neq a \circ b \circ c=(a \circ y)(x \circ c)$. By additivity the Euclidean inclusion holds for all of $\mathbf{A}$.

From part (ii) of the preceding lemma we can easily deduce the following result.

COROLlaRY 19. The residuated complex algebra of any small category is a Euclidean rm-algebra. 
In [Ma78] there is an example of a ur-algebra (in fact a weakly associative relation algebra) that is subdirectly irreducible but not simple. However, this example is not associative, which led B. Jónsson [J91a] to ask the question whether every subdirectly irreducible $r m$-algebra is simple. The example below shows that this is not the case, even restricted to Euclidean $r m$-algebras, hence the variety of all Euclidean $r m$-algebras is not a discriminator variety.

Recall that any quasiorder can be viewed as a category (the elements are the objects and the pairs $a \leq b$ are the morphisms). The Euclidean $r m$-algebras that arise in this way are in fact relative subalgebras of full relation algebras, relativised with respect to the quasiorder relation.

THEOREM 20. The complex algebra of the partial order of an infinite fence, viewed as a category, is a subdirectly irreducible (Euclidean) rm-algebra that is not simple.

Proof. Let $\mathbb{Z}$ be the set of integers and denote by $U$ the partial order of an infinite fence over $\mathbb{Z}$, i.e.

$$
U=\left\{(m, n) \in \mathbb{Z}^{2}: m=n \text { or }(m \text { is even and }|m-n|=1)\right\} .
$$

Let $\mathbf{A}$ be the residuated complex algebra of the structure $\left(U, \circ, \mathrm{id}_{\mathbb{Z}}\right)$, where $\circ$ is the partial operation

$$
(m, n) \circ(p, q)=(m, q) \quad \text { if } n=p \text { and }(m, q) \in U
$$

and $\operatorname{id}_{\mathbb{Z}}=\{(n, n): n \in \mathbb{Z}\}$. By Corollary 19, A is a Euclidean $r m$-algebra. Note that for any $(m, n) \in U$ we have

$$
\begin{aligned}
& 1 \triangleleft(m, n)= \begin{cases}\{(m, m)\} & \text { if } m \neq n \text { or } m \text { is even, } \\
\{(m, m),(m-1, m),(m+1, m)\} & \text { otherwise, }\end{cases} \\
& (m, n) \triangleright 1= \begin{cases}\{(n, n)\} & \text { if } m \neq n \text { or } n \text { is odd, } \\
\{(n, n),(n, n-1),(n, n+1)\} & \text { otherwise. }\end{cases}
\end{aligned}
$$

If we now let $a_{1}$ be any atom $(m, n)$ and define $a_{2 i}=\left(a_{2 i-1} \triangleright 1\right) \triangleright 1$ and $a_{2 i+1}=$ $1 \triangleleft\left(1 \triangleleft a_{2 i}\right)$ for $i=1,2,3, \ldots$, then the $a_{i}$ form an unbounded increasing sequence which eventually exceeds every atom.

Denoting the ideal of all finite joins of atoms of $\mathbf{A}$ by $I$, it follows that any nontrivial congruence ideal of $\mathbf{A}$ must contain $I$. On the other hand, $I$ is also a congruence ideal, hence it is the smallest nontrivial congruence ideal of $\mathbf{A}$. Therefore $\mathbf{A}$ is subdirectly irreducible but not simple.

\section{References}

[BP89] W. J. Blok and D. Pigozzi, On the structure of varieties with equationally definable principal congruences III, preprint, 1989.

[GM90] S. Ghilardi and G. C. Meloni, Modal logics with n-ary connectives, Z. Math. Logik Grundlag. Math. 36 (1990), 193-215. 
[J91] B. Jónsson, The preservation theorem for canonical extensions of Boolean algebras with operators, in: Proc. Birkhoff Sympos., to appear.

[J91a] —, Boolean algebras with operators, in: Proc. 1991 Summer Session "Algebras and order" at the Université de Montréal, Kluwer, to appear.

[JT51] B. Jónsson and A. Tarski, Boolean algebras with operators, Part I, Amer. J. Math. 73 (1951), 891-939.

[JTs91] B. Jónsson and C. Tsinakis, Relation algebras as residuated Boolean algebras, Algebra Universalis, to appear.

[Ma78] R. Maddux, Topics in relation algebras, doctoral dissertation, Univ. of California, Berkeley 1975.

[Ma82] —, Some varieties containing relation algebras, Trans. Amer. Math. Soc. 272 (2) (1982), 501-526.

[M75] R. McKenzie, On spectra, and the negative solution of the decision problem for identities having a finite nontrivial model, J. Symbolic Logic 40 (2) (1975), 186-196.

[MMT] R. N. McKenzie, G. F. McNulty and W. F. Taylor, Algebras, Lattices, Varieties, Volume I, The Wadsworth \& Brooks/Cole Mathematics Series, Wadsworth, Belmont, California, 1987.

[P61] W. Prenowitz, A contemporary approach to classical geometry, Amer. Math. Monthly 68 (No. 1, Part II) (1961)

[W78] H. Werner, Discriminator algebras, Stud. Algebra Anwendungen 6, Akademie-Verlag, Berlin 1978 